de fibres particulières que l'on ne distingue pas des IIB en histologie classique ;

- soit la technique d'identification des fibres IIB (ATPase acide classique) est imparfaite et ne permet pas de systématiquement bien typer les fibres musculaires.

Il est bien difficile de choisir entre ces deux hypothèses car on sait que certaines fibres existent mais ne sont pas détectables en histologie classique (cas des fibres IIx chez le porc). On sait aussi que la typologie myofibrillaire est dans un état dynamique (avec des possibili- tés de passage d'un type à l'autre) et qu'il peut donc aussi y avoir des fibres dans un état intermédiaire de façon transitoire et normale.

Quoiqu'il en soit, il paraît impossible d'essayer d'utiliser ces anticorps aujourd'hui en substitution des techniques ATPasiques classiques. De plus la perte totale de réactivité des anticorps S5 16D12, S5 8F3 et la diminution apparente de l'affinité du S4 17F7 sont encore un point d'interrogation supplémentaire sur leur spécificité réelle pour les types de fibres initialement identifiées.

\title{
Anticorps anti-chaînes lourdes de myosine : outils d'étude de la régénération musculaire dans l'espèce dinde
}

\section{Y. CHEREL, L. GUIGAND, M. WYERS}

INRA URA 703

Développement et Pathologie du tissu musculaire, École Nationale Vétérinaire, BP 40706, 44307 Nantes Cedex 03

\begin{abstract}
La production de dindes de souche médiumalourdie (mâles de 16 semaines abattus à $12 \mathrm{~kg}$ environ et femelles de 12 semaines abattues à $7 \mathrm{~kg}$ ) fournit des carcasses utilisées en découpe. Les produits issus de cette découpe ont des valeurs commerciales différentes : les pectoraux procurent une viande blanche (correspondant à des muscles rapides) transformée en escalopes ou rôtis, c'est la partie noble ; les cuisses et, dans une moindre mesure, les ailes sont composées de viande rouge, correspondant à des muscles mixtes rapides et lents. Ils sont à l'origine de steak, osso bucco et blanquette. Actuellement, la transformation de la viande pour fournir des produits de charcuterie est encore peu développée, même si cette transformation semble représenter un débouché d'avenir.
\end{abstract}

Les notions de qualité de la viande de dinde se résument aujourd'hui à deux problématiques différentes : la première concerne la couleur des muscles blancs (hétérogénéité de couleur ainsi que grisaillement) c'est-à-dire quasi-exclusivement les muscles pectoraux ; la seconde a trait à l'exsudation des viandes après transformation. Or, les muscles pectoraux sont des muscles homogènes rapides, formés presque totalement de fibres de type IIB. La production d'anticorps permettant de discriminer les chaînes lourdes de myosine (MHC) des types IIa et IIb dans le but de typer les fibres musculaires n'a donc pas, dans l'espèce dinde, l'intérêt qu'elle peut avoir en termes de qualité des viandes et de sélection dans d'autres espèces.

En revanche, cette production d'anticorps présente un intérêt en tant qu'outil d'étude de la biologie du tissu musculaire. En effet, pour l'étude de la croissance du tissu musculaire aussi bien que pour l'étude de sa régénération après lésion, l'expression des isoformes de myosine est un excellent marqueur de la différenciation cellulaire.

$\mathrm{Au}$ cours de la croissance, les fibres musculaires multinucléées fusionnent avec des myoblastes issus de la prolifération de cellules satellites. Le nombre de noyaux des fibres croît, ce qui permet, par l'intermédiaire des synthèses de myofilaments, d'augmenter la taille de la cellule, car le rapport nucléo-cytoplasmique est constant dans un type de fibre donné.

Après lésion de la fibre musculaire, les zones nécrosées sont éliminées par phagocytose macrophagique, puis les cellules satellites fournissent les myoblastes qui fusionnent avec la fibre blessée ou fusionnent entre eux pour fournir des néo-myotubes.

Après la fusion, ces néo-myotubes ou certaines portions de fibres matures synthétisent les protéines contractiles avec une succession d'isoformes qui reproduit le décours temporel observé pendant le développement embryonnaire. La mise en évidence de ces isoformes est un moyen d'étude de la différenciation cellulaire et de l'action de facteurs modulant le développement du tissu.

Nous utilisons au laboratoire, en immunofluorescence ou en immunohistoenzymologie, plusieurs anticorps anti-isomyosines développementales dans le cadre de travaux sur la régénération musculaire, et en immunocytochimie sur culture de cellules satellites pour caractériser les populations de cellules. La possession d'anticorps anti-MHC IIa et IIb compléterait de façon très intéressante la gamme existante. 


\section{Conditions expérimentales}

Les anticorps monoclonaux anti-MHC produits dans le cadre du projet Noé ont tous été testés sur différents muscles de dinde par immunofluorescence : les coupes réalisées au cryostat ont été fixées à l'acétone à $4{ }^{\circ} \mathrm{C}$ pendant 30 minutes puis incubées avec l'anticorps primaire dilué au $1 / 10$ et $2 \%$ de BSA pendant 1 heure à $37{ }^{\circ} \mathrm{C}$ puis lavés dans du PBS pH 7,2. Les coupes ont ensuite été incubées à la température de la pièce avec l'anticorps secondaire lié au FITC (Euromedex AP $160 \mathrm{~F})$ dilué au $1 / 100$ pendant 1 heure. Lorsque le signal paraissait prometteur, un contrôle à une dilution de l'anticorps primaire à $1 / 100$ a été réalisé.

Afin de fixer avec précision le type de chaque anticorps, chacun d'entre eux a été utilisé sur 4 muscles coupés en congélation : le pectoral (pectoralis major), composé presque exclusivement de fibres de type IIB, le PLD (posterior latissimus dorsi), composé d'un mélange de fibres de type IIA et IIB, l'ITC (iliotibialis cranialis), formé d'une zone hétérogène composée de fibres de type I, IIA et IIB et d'une zone hétérogène composée de fibres de type IIA et IIB, et l'ALD (anterior latissimus dorsi), composé de fibres de type III.

\section{Résultats}

Les résultats suivants ont été obtenus :

- 1 anticorps a fourni un marquage très faible des fibres squelettiques mais il marquait très fortement les artères présentes sur la coupe (MHC spécifique de fibre musculaire lisse) ;
- 32 anticorps ont fourni un signal nul ou faible ;

- 1 anticorps un signal très faible sur des fibres de type III (lentes) ;

- 16 anticorps un signal positif sur les fibres de type II, sans les différencier ;

- 5 anticorps pan-myosine (c'est-à-dire couvrant toutes les isoformes de myosine), marquage de toutes les fibres ;

- 3 pan-myosine avec un signal supérieur pour les fibres de type I (lentes);

- 3 pan-myosine avec un signal supérieur pour les fibres de type II (rapides) ;

- 3 anticorps ont fourni un signal restreint aux fibres de type II, mais en se fixant de façon plus marquée sur certaines fibres II et beaucoup plus faible sur d'autres fibres II, aboutissant à la formation d'une mosaïque à l'observation des coupes transversales.

Ces trois derniers anticorps, à savoir S5 8H2, S5 7D4 et S5 11D6, ainsi que l'anticorps anti-I S5 7F10 ont été retenus.

L'analyse en Western-blot a montré que deux des anticorps anti-myosine rapide fournissant une mosaïque en immunofluorescence étaient des anticorps anti-II, et que le troisième était un anticorps anti-IIa, mais la mosaïque observée ne correspond pas à une distribution de fibres de type IIA et IIB, des fibres IIB étant positives. On peut interpréter cette distorsion par l'existence de fibres contenant à la fois des isoformes IIa et IIb correspondant à une maturation incomplète.

Les anticorps générés par ce projet constituent des outils utilisables en immunohistochimie. Ils sont en cours de test sur des myotubes issus de la régénération musculaire in vivo et sur des myoblastes en culture.

\section{E. BARREY, \\ J. P. VALETTE, M. JOUGLIN*}

\section{INRA Station de Génétique Quantitative et Appliquée, 78352 Jouy-en-Josas \\ * Ecole Nationale Vétérinaire d'Alfort, Laboratoire de Biomécanique, 94704 Maisons- Alfort Cedex}

\section{Analyse de la composition en chaînes lourdes de myosine chez le cheval : application à la sélection du cheval de course}

La performance du cheval de course dépend de plusieurs facteurs biologiques interdépendants : la respiration, la circulation sanguine, le métabolisme énergétique, les contractions musculaires, la locomotion et l'aptitude comportementale. Les propriétés contractiles et métaboliques des muscles propulseurs constituent l'un des facteurs limitants qui déterminent la force et l'endurance du cheval à la course (Barrey 1994). La force développée par les contractions musculaires dépend à la fois du nombre total et du type de fibres qui composent le muscle. Chez le cheval, il existe trois isoformes de la chaîne lourde de la myosine : la myosine lente (MHC I), et deux myosines rapides (MHC IIa et $\mathrm{MHC} \mathrm{IIb}$ ). Les fibres musculaires très riches en isoforme MHC IIb produisent des contractions plus puissantes mais moins durables. Une fibre musculaire peut être composée de plusieurs isoformes de la myosine (I+IIa ou IIa+IIb) et la myosine dominante détermine le type de fibre parmi les types lent (I) ou rapides (IIA, IIB) lorsqu'on les observe en histologie par la méthode de coloration ATPase (Rivero et al 1996). Le type de fibre rapide dénommée IIC en histolo- 